\title{
MAGIC detection of very high energy $\gamma$-ray emission from the low-luminosity blazar 1ES 1741+196
}

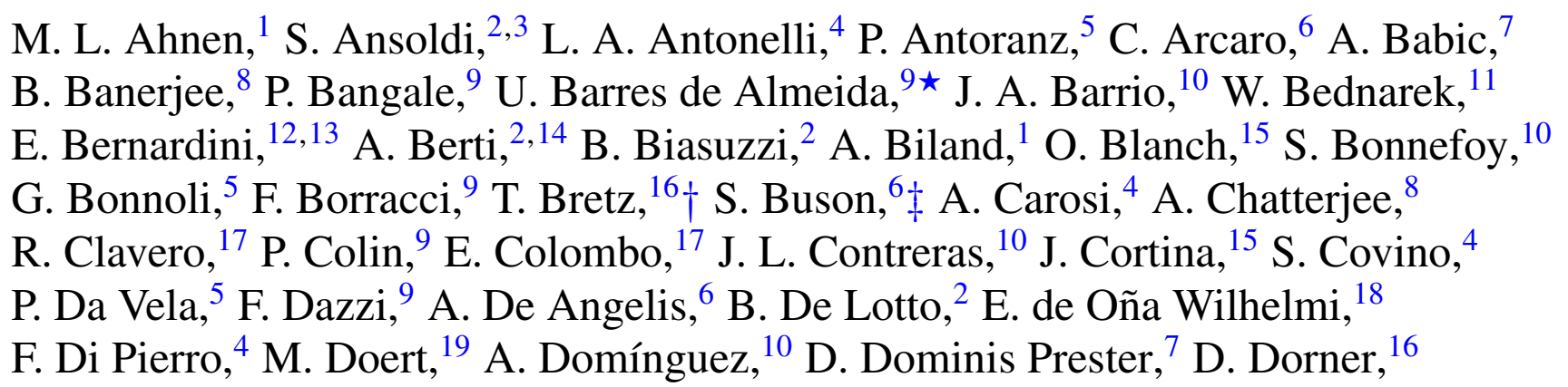
M. Doro, ${ }^{6}$ S. Einecke, ${ }^{19}$ D. Eisenacher Glawion, ${ }^{16}$ D. Elsaesser, ${ }^{19}$ M. Engelkemeier, ${ }^{19}$ V. Fallah Ramazani, ${ }^{20}$ A. Fernández-Barral, ${ }^{15}$ D. Fidalgo,${ }^{10} \mathrm{M}$. V. Fonseca, ${ }^{10}$ L. Font ${ }^{21}$ K. Frantzen, ${ }^{19}$ C. Fruck, ${ }^{9}$ D. Galindo, ${ }^{22}$ R. J. García López, ${ }^{17}$ M. Garczarczyk, ${ }^{12}$ D. Garrido Terrats, ${ }^{21}$ M. Gaug, ${ }^{21}$ P. Giammaria, ${ }^{4}$ N. Godinović, ${ }^{7}$ D. Gora, ${ }^{12}$ D. Guberman, ${ }^{15}$ D. Hadasch, ${ }^{23}$ A. Hahn, ${ }^{9}$ M. Hayashida, ${ }^{23}$ J. Herrera, ${ }^{17}$ J. Hose, ${ }^{9}$ D. Hrupec,${ }^{7}$ G. Hughes,${ }^{1}$ W. Idec, ${ }^{11}$ K. Kodani, ${ }^{23}$ Y. Konno, ${ }^{23}$ H. Kubo, ${ }^{23}$ J. Kushida, ${ }^{23}$ A. La Barbera, ${ }^{4}$ D. Lelas,${ }^{7}$ E. Lindfors,${ }^{20}$ S. Lombardi, ${ }^{3}$ F. Longo, ${ }^{2,14}$ M. López, ${ }^{10}$ R. López-Coto, ${ }^{15} \S$ P. Majumdar, ${ }^{8}$ M. Makariev, ${ }^{24}$ K. Mallot, ${ }^{12}$ G. Maneva, ${ }^{24}$ M. Manganaro, ${ }^{17}$ N. Mankuzhiyil, ${ }^{2} \boldsymbol{\top} \|$ K. Mannheim, ${ }^{16}$ L. Maraschi, ${ }^{4}$ B. Marcote, ${ }^{22}$ M. Mariotti, ${ }^{6}$ M. Martínez, ${ }^{15}$ D. Mazin,,${ }^{9,25}$ U. Menzel, ${ }^{9}$ J. M. Miranda,${ }^{5}$ R. Mirzoyan, ${ }^{9}$ A. Moralejo, ${ }^{15}$ E. Moretti, ${ }^{9}$ D. Nakajima, ${ }^{23}$ V. Neustroev, ${ }^{20}$ A. Niedzwiecki, ${ }^{11}$ M. Nievas Rosillo, ${ }^{10}$ K. Nilsson, ${ }^{20} \#$ K. Nishijima, ${ }^{23}$ K. Noda, ${ }^{9}$ L. Nogués ${ }^{15}$ S. Paiano, ${ }^{6}$ J. Palacio, ${ }^{15}$ M. Palatiello, ${ }^{2}$ D. Paneque, ${ }^{9}$ R. Paoletti, ${ }^{5}$ J. M. Paredes, ${ }^{22}$ X. Paredes-Fortuny, ${ }^{22}$ G. Pedaletti, ${ }^{12}$ M. Peresano, ${ }^{2}$ L. Perri, ${ }^{4}$ M. Persic, ${ }^{2,26}$ J. Poutanen, ${ }^{20}$ P. G. Prada Moroni, ${ }^{27}$ E. Prandini, ${ }^{1,28}$ I. Puljak, ${ }^{7}$ J. R. Garcia, ${ }^{9}$ I. Reichardt, ${ }^{6}$ W. Rhode,${ }^{19}$ M. Ribó, ${ }^{22}$ J. Rico, ${ }^{15}$ T. Saito, ${ }^{23}$ K. Satalecka, ${ }^{12}$ S. Schroeder, ${ }^{19}$ T. Schweizer, ${ }^{9}$ S. N. Shore, ${ }^{27}$ A. Sillanpää, ${ }^{20}$ J. Sitarek, ${ }^{11}$ I. Snidaric, ${ }^{7}$ D. Sobczynska, ${ }^{11}$ A. Stamerra, ${ }^{4}$ M. Strzys, ${ }^{9}$ T. Surić, ${ }^{7}$ L. Takalo,${ }^{20}$ H. Takami,${ }^{23 \star \star}$ F. Tavecchio, ${ }^{4}$ P. Temnikov, ${ }^{24}$ T. Terzić, ${ }^{7}$ D. Tescaro, ${ }^{6}$ M. Teshima,,${ }^{9,25}$ D. F. Torres,${ }^{29}$ T. Toyama, ${ }^{9}$ A. Treves,${ }^{2}$ G. Vanzo, ${ }^{17}$ V. Verguilov, ${ }^{24}$ I. Vovk, ${ }^{9}$ J. E. Ward, ${ }^{15}$ M. Will,,${ }^{17}$ M. H. Wu, ${ }^{18}$ R. Zanin, ${ }^{22} \dagger \dagger$ (MAGIC

\footnotetext{
* Present address: Centro Brasileiro de Pesquisas Físicas (CBPF/MCTI), R. Dr. Xavier Sigaud, 150 - Urca, Rio de Janeiro - RJ, 22290-180, Brazil. $\dagger$ Present address: Ecole polytechnique fédérale de Lausanne (EPFL), Lausanne, Switzerland.

$\ddagger$ Present addresses: NASA Goddard Space Flight Center, Greenbelt, MD 20771, USA, Department of Physics, Department of Astronomy, University of Maryland, College Park, MD 20742, USA.

$\S$ Present address: Max-Planck-Institut fur Kernphysik, PO Box 103980, D-69029 Heidelberg, Germany.

T Present address: Astrophysical Sciences Division, BARC, Mumbai, India.

|| E-mail: mankuzhiyil.nijil@gmail.com

\# Present address: Finnish Centre for Astronomy with ESO (FINCA), Turku, Finland.

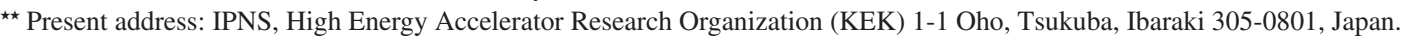

$\dagger \dagger$ Present address: Max-Planck-Institut fur Kernphysik, PO Box 103980, D-69029 Heidelberg, Germany.

$\ddagger \ddagger$ Present addresses: NASA Goddard Space Flight Center, Greenbelt, MD 20771, USA, Department of Physics, Department of Astronomy, University of Maryland, College Park, MD 20742, USA.
} 


\title{
collaboration), J. Becerra González, ${ }^{17} \ddagger \ddagger$ B. Rani, ${ }^{30}$ (Fermi-LAT collaboration), F. Krauss ${ }^{31}$ M. Perri,${ }^{4,32}$ F. Verrecchia ${ }^{4,32}$ and R. Reinthal ${ }^{20}$ \\ Affiliations are listed at the end of the paper
}

Accepted 2017 February 21. Received 2017 February 21; in original form 2016 October 31

\begin{abstract}
We present the first detection of the nearby $(z=0.084)$ low-luminosity BL Lac object $1 \mathrm{ES} 1741+196$ in the very high energy $(E>100 \mathrm{GeV})$ band. This object lies in a triplet of interacting galaxies. Early predictions had suggested 1ES 1741+196 to be, along with several other high-frequency BL Lac sources, within the reach of MAGIC detectability. Its detection by MAGIC, later confirmed by VERITAS, helps to expand the small population of known TeV BL Lacs. The source was observed with the MAGIC telescopes between 2010 April and 2011 May, collecting $46 \mathrm{~h}$ of good quality data. These observations led to the detection of the source at $6.0 \sigma$ confidence level, with a steady flux $F(>100 \mathrm{GeV})=\left(6.4 \pm 1.7_{\text {stat }} \pm\right.$ $\left.2.6_{\text {syst }}\right) \times 10^{-12} \mathrm{ph} \mathrm{cm}^{-2} \mathrm{~s}^{-1}$ and a differential spectral photon index $\Gamma=2.4 \pm 0.2_{\text {stat }} \pm 0.2_{\text {syst }}$ in the range of $\sim 80 \mathrm{GeV}-3 \mathrm{TeV}$. To study the broad-band spectral energy distribution (SED) simultaneous with MAGIC observations, we use KVA, Swift/UVOT and XRT and Fermi/LAT data. One-zone synchrotron-self-Compton (SSC) modelling of the SED of 1ES 1741+196 suggests values for the SSC parameters that are quite common among known TeV BL Lacs except for a relatively low Doppler factor and slope of electron energy distribution. A thermal feature seen in the SED is well matched by a giant elliptical's template. This appears to be the signature of thermal emission from the host galaxy, which is clearly resolved in optical observations.
\end{abstract}

Key words: BL Lacertae objects: individual: (1ES 1741+196)-gamma-rays: galaxies.

\section{INTRODUCTION}

Blazars are thought to be black hole $(\mathrm{BH})$ powered active galactic nuclei (AGNs) whose relativistic jets are closely aligned with our line of sight. They constitute the most numerous class of detected extragalactic very high energy (VHE: $E>100 \mathrm{GeV}) \gamma$-ray sources. Their spectral energy distribution (SED) typically shows two emission components: (i) one component peaks at $\mathrm{eV}-\mathrm{keV}$ energies, interpreted as synchrotron radiation emitted by relativistic electrons moving in the jet's magnetic field; and (ii) another component, which peaks at $\gamma$-ray frequencies, commonly interpreted as arising from inverse Compton (IC) scattering of lower energy photons (Rees 1967) - the latter being either the above-mentioned synchrotron photons internal to the jet [Synchro-Self-Compton (SSC) scenario, see Maraschi, Ghisellini \& Celotti 1992] or some other photon field external to the jet (External Compton scenario, see Dermer \& Schlickeiser 1993). The high energy peak may also result from hadronic processes, as proposed by Mannheim (1993). BL Lac objects are blazars characterized by a featureless, highly polarized, broad-band (radio to VHE) continuum emission.

1ES $1741+196$ is a high-frequency-peaked BL Lac object (HBL; where the synchrotron peaks at X-ray, while its IC counterpart peaks at VHE), at coordinates (J2000) RA $=17: 43: 57.8$ (hh:mm:ss) and Dec. $=19: 35: 09$ (dd:mm:ss), at redshift $z=0.084$. Its host galaxy is one of the most luminous and largest $\left(M_{R}=-24.85 ; r_{e}=51.2 \mathrm{kpc}\right)$ among BL Lac host galaxies. Two nearby (7.2 and $25.2 \mathrm{kpc})$ companion galaxies at the same redshift suggest that 1ES $1741+196$ could be a BL Lac object in a triplet of interacting galaxies (Heidt et al. 1999). It was detected in radio, optical, X-ray and high en- ergy (HE: $E>100 \mathrm{MeV}$ ) $\gamma$-ray frequencies (Rector, Cabzuda \& Stocke 2003; Heidt et al. 1999; Padovani \& Giommi 1995; Acero et al. 2015). Its high resolution radio map (Piner \& Edwards 2014) shows a parsec scale one-sided jet. The jet-counterjet brightness ratio suggests a Doppler factor of $\delta>4$, for a viewing angle of a few degrees.

Prompted by the prediction of TeV flux based on the BeppoSAX observations and the SSC model (Costamante \& Ghisellini 2002), MAGIC observed this source in mono-mode for a total of $16 \mathrm{~h}$ between 2007 July and 2008 August, obtaining a significance of $2 \sigma$, and a flux upper limit of $F\left(3.6 \times 10^{-11} \mathrm{ph} \mathrm{cm}^{-2} \mathrm{~s}^{-1}\right.$ (Aleksić et al. 2011). Further MAGIC observations carried out between 2010 April and 2011 May in stereoscopic mode finally led to the detection of the source at VHE $\gamma$-ray frequencies (Berger et al. 2011). This was later confirmed by VERITAS (Abeysekara et al. 2016).

In this paper, we study the emission features of the 1ES $1741+196$, the only BL Lac object detected in a triplet of interacting galaxies, using the data collected from MAGIC and other multifrequency instruments. In Section 2, we describe the multi frequency data used for this analysis. The results are presented in Section 3, discussed in Section 4 and summarized in Section 5.

\section{OBSERVATIONS AND DATA ANALYSIS}

Observations of 1ES 1741+196 during time periods that include the MAGIC observation window were performed in the optical, X-ray and $\mathrm{HE} \gamma$-ray ranges, which are discussed in detail in the following sections. 


\subsection{KVA}

The KVA (Kungliga Vetenskapsakademien Academy) ${ }^{1}$ telescopes are located at La Palma but operated remotely by the Tuorla Observatory in Finland. These telescopes are used mainly for optical support observations for the MAGIC telescopes. The KVA telescopes consist of a $60-\mathrm{cm}$ telescope that is used for polarimetric observations and a $35-\mathrm{cm}$ telescope used for photometry simultaneous with MAGIC observations. Furthermore, the smaller $35-\mathrm{cm}$ telescope monitors potential VHE $\gamma$-ray candidate AGNs in order to trigger MAGIC observations if one of these selected objects is in a high optical state. These observations are performed in the $R$ band and the magnitude of the source is measured from CCD images using differential photometry, i.e. by comparing the brightness of the object with that of several calibrated stars in the same field of view. The data were processed by the reduction programmes developed in Tuorla Observatory (see Nilsson et al., in preparation, and the references therein).

\subsection{Swift}

The Swift satellite, which was launched in 2004 (Gehrels et al. 2004) carries three instruments: the Burst Alert Telescope (BAT; sensitive 15-150 keV; Barthelemy et al. 2005), the X-ray telescope (XRT; sensitive 0.2-10 keV; Burrows et al. 2005) and the UV/Optical Telescope (UVOT; sensitive $170-600 \mathrm{~nm}$; Roming et al. 2005).

The Swift/XRT data that fall in the MAGIC observation period were taken in photon counting mode on 2010 July 30 and 2011 January 21 . These data were processed by the XRTPIPELINE (version 0.13.1) distributed by HEASARC within the HEASOFT package (v.6.16) using standard procedure. Events with grades 0-12 were selected (see Burrows et al. 2005) and the response matrices available in the Swift CALDB (20110101v014) were used. The source events in the $0.3-10 \mathrm{keV}$ range within a circle with a radius of $22 \operatorname{arcsec}$ were selected for the spectral analysis. The background was extracted from off-source circular regions of the same radius. The spectra were extracted from the corresponding event files and binned using GRPPHA to ensure a minimum of 20 counts per energy bin. Spectral analysis was performed using XSPEC version 12.8.2.

Swift/UVOT source counts were extracted from a circular region of radius 5 arcsec, centred on the source position. The background was estimated from three circular source free regions of the same radius. These data were processed with the UVOTMAGHIST task of the HEASOFT package.

\section{$2.3 \mathrm{Fermi} / \mathrm{LAT}$}

The pair-conversion Large Area Telescope (LAT) on board the Fermi satellite monitors the $\gamma$-ray sky in survey mode every $3 \mathrm{~h}$ in the energy range from $20 \mathrm{MeV}$ to $>300 \mathrm{GeV}$ (Atwood et al. 2009). The data presented in this paper were selected from a $15^{\circ}$ radius region of interest (ROI) centred at the location of the 1ES 1741+196, during the first $6.7 \mathrm{yr}$ of the mission from 2008 August 4 to 2015 April 7 (MJD 54682.7-57119.3). We analysed the data in the energy range from $100 \mathrm{MeV}$ to $300 \mathrm{GeV}$. The analysis was performed with the SCIENCETOOLS software package version v9r33p0 and the instrument response function P7REP_SOURCE_V15 (Ackermann et al. 2012). The event selection was based on Pass 7 reprocessed source class events, and a zenith angle cut of $<100^{\circ}$ was applied to reduce the contamination from the Earth limb. The Galactic diffuse emission model (Acero et al. 2016) and isotropic component used were gll_iem_v05_rev1.fit and iso_source_v05.txt, respectively as recommended for Pass 7 Reprocessed Source event class. ${ }^{2}$ The normalizations of both components in the background model were allowed to vary freely during the spectral fitting. A binned maximumlikelihood method analysis was used (Mattox et al. 1996).

For a first likelihood fit making use of GTLIKE, the model includes all the sources within $20^{\circ}$ of the source of interest that are included in the Fermi/LAT third source catalog (Acero et al. 2015). For the spectral fit (simple power law), spectral indices and fluxes were left free for the sources within $15^{\circ}$, while sources from $15^{\circ}$ to $20^{\circ}$ were frozen to the catalog value. From the residual of the model (created using GTMODEL) with respect to the data within the ROI, in addition to the 3FGL sources, we identified one new source with test statistic $\mathrm{TS}=26.1$ located $9.9^{\circ}$ from 1ES $1741+196$. This was included in the model. In addition, five more sources with TS between 5.3 and 12.8 and located between $9.1^{\circ}$ and $11.9^{\circ}$ from 1 ES $1741+196$ were included in the model. The best location of these six additional sources were found using GTFINDSRC. The sources with TS $<5$ were deleted from the model. A second maximum-likelihood analysis was performed on the updated source model. For the light-curve calculation in $1 \mathrm{yr}$ time bins shown in Fig. 2, only the source of interest and the diffuse models were left free to vary, while the rest of the sources considered in the analysis were fixed to the values obtained from the analysis of the entire data sample. Also variability on monthly time-scales was investigated.

\subsection{MAGIC}

MAGIC is a system of two 17-m dish Imaging Atmospheric Cherenkov Telescopes (IACT) located at the Roque de los Muchachos Observatory $\left(28.8^{\circ} \mathrm{N}, 17.8^{\circ} \mathrm{W}, 2200 \mathrm{~m}\right.$ a.s.1. $)$, on the Canary Island of La Palma, Spain. Since 2009, the MAGIC telescopes operate stereoscopically, with a sensitivity of $<0.7$ percent crabunit (integrated flux from the Crab nebula) for energies $>220 \mathrm{GeV}$ in $50 \mathrm{~h}$ of observations (Aleksić et al. 2012).

The MAGIC telescopes observed 1ES 1741+196 for 53 nights from 2010 April 10 until 2011 May 26, for a total observation time of approximately $57 \mathrm{~h}$ in the so-called wobble mode (Fomin et al. 1994). The data were taken for zenith angles in the range of $9^{\circ}-38^{\circ}$, which resulted in an energy threshold [defined as the peak of the Monte Carlo (MC) simulated photon energy distribution for a Crab-Nebula-like spectrum after all analysis cuts] of $90 \mathrm{GeV}$.

After the application of standard quality checks based on the rate of the stereo events and the distributions of basic image parameters, $\sim 46 \mathrm{~h}$ of effective on-time data were selected. Data analysis was performed using the standard software package MARS (Albert et al. 2008a; Aliu et al. 2009), including the latest routines for stereoscopic analysis (Lombardi et al. 2011; Zanin et al. 2011; Aleksić et al. 2012). After the calibration (Albert et al. 2008b) and image cleaning of the events recorded by each telescope, the information coming from the individual telescopes is combined and the calculation of basic stereo image parameters is performed. For $\gamma /$ hadron separation and the $\gamma$-direction estimation, a multivariate method called RANDOM FOREST (Albert et al. 2008c) was applied using image parameters (Hillas 1985), timing information (Aliu et al. 2009) and stereo parameters (Aleksić et al. 2012), to compute a $\gamma /$ hadron discriminator, called hadronness. While computing the significance

${ }^{1}$ Tuorla Blazar monitoring program, http://users.utu.fi/kani.

${ }^{2}$ http://fermi.gsfc.nasa.gov/ssc/data/access/lat/BackgroundModels.html 


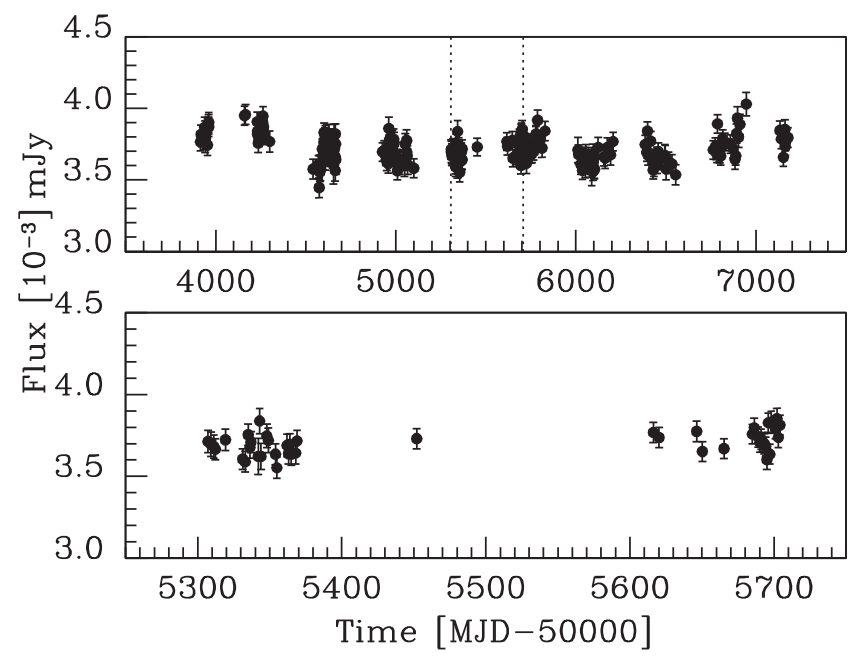

Figure 1. Optical $R$-band light curve from $7 \mathrm{yr}$ of monitoring observations performed by the Tuorla Observatory. The contribution of the host galaxy has not been subtracted. The MAGIC observation window in 2010-2011 is indicated by the vertical lines in the top panel whereas the bottom panel presents the zoomed light curve in the MAGIC observation period.

of the signal coming from the 1ES $1741+196$ sky region, we applied single cuts in hadronness and $\theta^{2},{ }^{3}$ which were optimized to maximize the significance of the signal (above $250 \mathrm{GeV}$ ) in a Crab nebula data set. Conversely, in deriving the spectrum and the light curve of the source, we applied different cut values in hadronness that, for each logarithmic energy bin, yield a gamma efficiency of 90 per cent in the MC gamma data set. These procedures, which are regularly used to analyse MAGIC data, are described in detail in Aleksić et al. (2012).

\section{RESULTS}

In the following sections, the analysis results from the optical, $\mathrm{X}$-ray, HE and VHE data are presented.

\subsection{KVA}

The top panel of Fig. 1, shows the light curve obtained from the photometric observations of KVA, between 2006 June and 2013 November, while the bottom panel shows the light curve from 2010 April 21 to 2011 May 23 that coincides with the MAGIC observation window.

The brightness was corrected for the dust in the Galaxy (Schlegel, Finkbeiner \& Davis 1998). Since the host galaxy belongs to a triplet of interacting galaxies, we used a slightly different approach to estimate the host galaxy magnitude, using a Nordic Optical Telescope (NOT) image (see also Nilsson et al. 2007). After subtracting the central point source based on the scaling from the model fit and convolving the image with a seeing of 2 arcsec (typical KVA good seeing value), the counts inside a radius of $7.5 \operatorname{arcsec}$ (which is the KVA measurement aperture) were measured. In order to match this NOT image to the same calibration used in the case of KVA monitoring, the brightness of two unsaturated stars in the NOT image (convolved with 2 arcsec seeing) were calculated. In order to derive the transformation from counts to magnitude, we measured the

\footnotetext{
${ }^{3}$ The parameter $\theta^{2}$ is the squared angular distance between the reconstructed source position of the events and the nominal position of the expected source.
}

same stars (as in the NOT image) using the KVA data with the same reference star that is used in the monitoring. Using this approach, the host magnitude has been computed. The flux of the host galaxy turned out to be $2.5 \pm 0.3 \mathrm{mJy}$.

After subtracting the estimated host galaxy flux from the observed KVA flux of 1ES 1741+196 (averaged over the MAGIC observation; Fig. 1), the residual KVA flux - an average value attributable to the nuclear region of blazar over the MAGIC observation time - is $(1.06 \pm 0.01) \mathrm{mJy}$. This matches the emission level monitored over the whole 7-yr span of KVA observations, i.e. (1.07 \pm $0.01) \mathrm{mJy}$. The source shows only marginal variability during the 7-yr KVA survey (as also shown in Lindfors et al. 2016), and hardly any variability during the MAGIC observations (respectively, top and bottom panels of Fig. 1).

\subsection{Swift}

The X-ray spectra can be well described by a simple power law $\left(\chi^{2} /\right.$ d.o.f $\left.=1.1\right)$ in the range of $0.3-10 \mathrm{keV}$, with a photon index $\Gamma=1.9 \pm 0.1$, and a normalization constant $f_{0}=(2.8 \pm 0.1) \times$ $10^{-3} \mathrm{keV}^{-1} \mathrm{~cm}^{-2} \mathrm{~s}^{-1}$ at $1 \mathrm{keV}$. The neutral hydrogen-equivalent column density was fixed to the Galactic value in the direction of the source, which is $7.36 \times 10^{20} \mathrm{~cm}^{-2}$ (Kalberla et al. 2005). We have also found that there is no significant spectral variability in the XRT observations of 2010 July 30 and 2011 January 21.

The fluxes obtained from Swift/UVOT analysis have been corrected for Galactic extinction $E(B-V)=0.079$ mag (Schlegel et al. 1998). The exact amount of the host galaxy contribution is not given in the literature. Hence, we estimated the host galaxy magnitude $V=1.1 \mathrm{mJy}, B=0.5 \mathrm{mJy}$ and $U=0.1 \mathrm{mJy}$ based on the $R$-band value (aperture 5 arcsec) from Nilsson et al. (2007) by using galaxy colours at $z=0$ (Fukugita, Shimasaku \& Ichikawa 1995). These derived values dominate the measured fluxes. Since Fukugita et al. (1995) does not provide the error estimate in the above filters, we roughly estimated the error in the $V, B$ and $U$ bands (which is $\sim 0.3 \mathrm{mJy})$, by taking into account the error in $R$-band $(0.26 \mathrm{mJy})$. The estimated error in $B$ and $U$ bands are comparable to, or larger than, the estimated host galaxy flux. Considering the rather large host galaxy magnitude (compared to the measured flux) and the high uncertainty, the fluxes in these bands will not be considered in the (non-thermal) SED modelling in this paper. Indeed, in Section 4, we will see that the Swift/UVOT data can be nicely interpreted as arising from the thermal emission of the elliptical host galaxy.

\subsection{Fermi/LAT}

In Fig. 2, the Fermi/LAT fluxes and spectral indices of 1ES $1741+196$ are plotted as a function of time in bins of $1 \mathrm{yr}$. Slight hints of variability, especially in the spectral index, do exist with $\chi_{v}^{2}=8.1 / 5$ and $11.46 / 5$ for, respectively, the flux and the spectral index, while fitting with a constant line. Since the source was not bright enough, variability on shorter time-scales cannot be investigated. Also, no month-scale variability was found, compatible with previous claims in the 3FGL catalog (Acero et al. 2015 with a variability index of 38.3).

We have analysed HE Fermi/LAT data contemporaneous to the MAGIC observations. The LAT data were collected from 2010 April 10 (MJD 55296) to 2011 May 26 (MJD 55707). A point like source positionally consistent with 1 ES $1741+196$ was detected with a TS $=19.4(\sim 4.4 \sigma)$. The best-fitting parameters for the model result in a spectral index of $\Gamma=1.6 \pm 0.1$ and an integral flux $F$ $(E>100 \mathrm{MeV})=(2.0 \pm 0.4) \times 10^{-9} \mathrm{ph} \mathrm{cm}^{-2} \mathrm{~s}^{-1}$. The spectral 


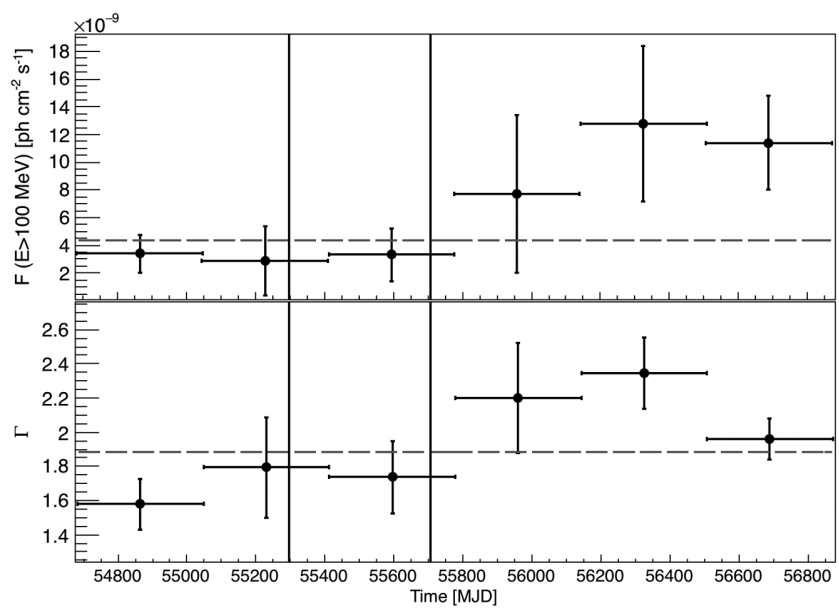

Figure 2. 1-yr binning Fermi/LAT light curve for $E>100 \mathrm{MeV}$ (top) and evolution of the spectral index as a function of the time (bottom). The grey dashed lines represent the mean flux and spectral index during the first $6.7 \mathrm{yr}$ of the mission, respectively. The period between the vertical bars denotes the MAGIC observation window.

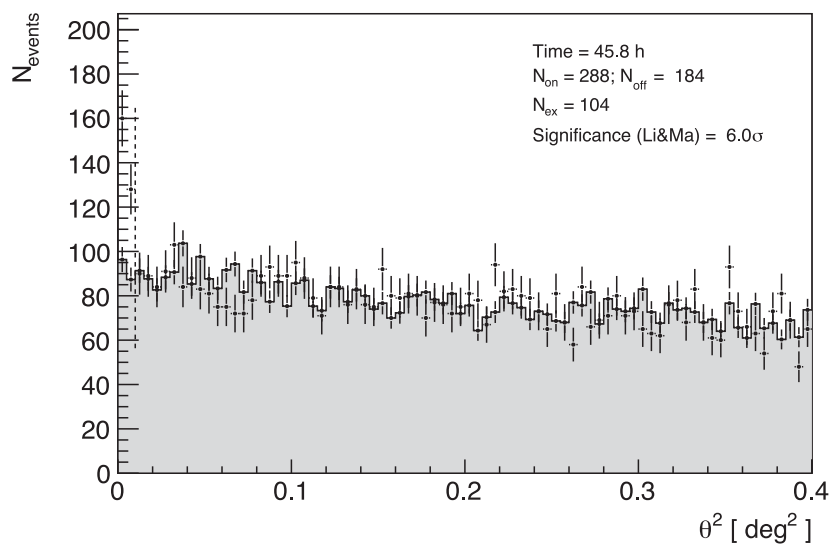

Figure 3. $\theta^{2}$ distributions of the 1 ES $1741+196$ signal and background estimation from $46 \mathrm{~h}$ of MAGIC stereo observations for $E>250 \mathrm{GeV}$. The region between zero and the vertical dashed line (at $0.01 \mathrm{deg}^{2}$ ) represents the signal region.

index reported in the 3FGL (Acero et al. 2015) is $1.8 \pm 0.1$, while the one reported in 1FHL (Ackermann et al. 2013) is $2.1 \pm 0.5$. For comparison purposes, a spectrum has also been produced for the whole data sample collected by the LAT from 2008 August 4 to 2015 April 7 (MJD 54682.7-57119.3) above $100 \mathrm{MeV}$ using the same procedure.

\subsection{MAGIC}

The $\gamma$-ray signal from the source is estimated from the so-called $\theta^{2}$ plot, after the application of energy-dependent cuts to events (including hadronness), and within a fiducial $\theta^{2}$ signal region. In order to evaluate the residual background of the observation, the $\theta^{2}$ distribution around a nominal background control region is also calculated. Fig. 3 shows the $\theta^{2}$ distribution of the events. We found an excess of $104 \pm 8$ events in the fiducial signal region with $\theta^{2}<0.01 \mathrm{deg}^{2}$, corresponding to a significance of $6.0 \sigma \mathrm{Li} \& \mathrm{Ma}(1983)$.

Fig. 4 shows the light curve of the source with a time binning of $28 \mathrm{~d}$, considering the length of a moon-cycle, which determines the observational season of IACTs. During the observation period

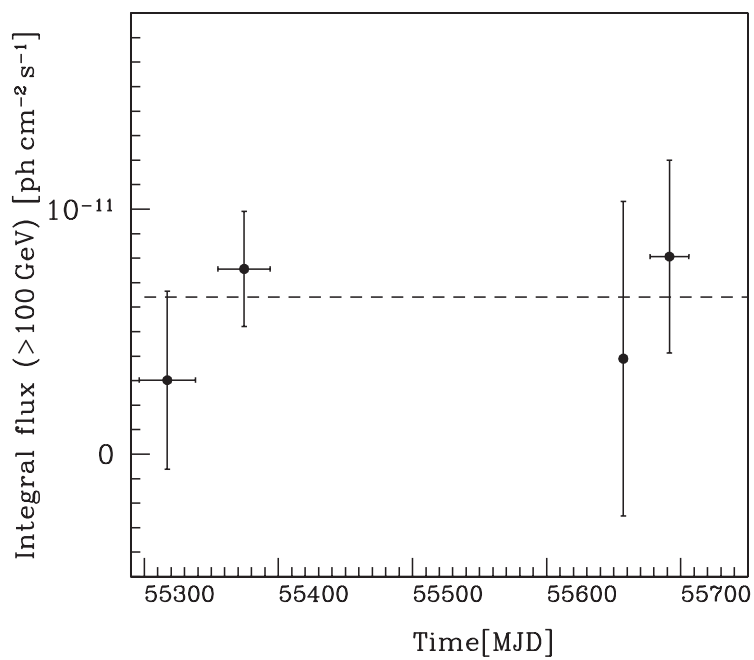

Figure 4. 1ES 1741+196 light curve during MAGIC observation period in a time bin of $28 \mathrm{~d}$. The horizontal line represents the fit to the data assuming a constant flux (see text for details).

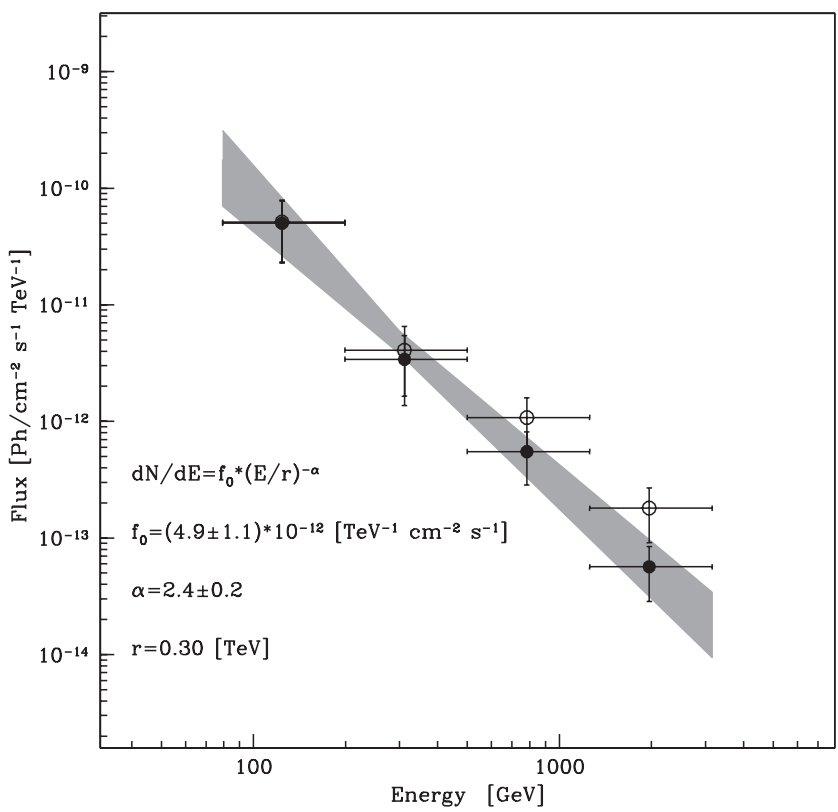

Figure 5. 1ES 1741+196 differential energy spectrum measured by MAGIC (filled circle), and EBL corrected spectrum using the Franceschini et al. (2008) model (empty circle). The fit function is shown as a grey bow-tie.

no significant variability was detected. The light curve can be fitted with a constant flux hypothesis of $(6.4 \pm 1.7) \times 10^{-12} \mathrm{ph} \mathrm{cm}^{-2} \mathrm{~s}^{-1}$ with a $\chi^{2} /$ d.o.f. $=1.4 / 3$.

Fig. 5 shows the spectral points of $1 \mathrm{ES} 1741+196$, which are obtained by the Bertero unfolding method (Albert et al. 2007). We also show the spectral points after correcting the extragalactic background light (EBL) using the Franceschini, Rodighiero \& Vaccari (2008) model. The bow-tie shows the power law fit obtained with the forward-folding method. The spectrum in the range $\sim 80 \mathrm{GeV}<E<3 \mathrm{TeV}$ can be well described by a simple power law $\left(\chi^{2} /\right.$ d.o.f. $\left.=2.16 / 6\right)$ :

$\frac{\mathrm{d} N}{\mathrm{~d} E}=f_{0}\left(\frac{E}{0.3 \mathrm{TeV}}\right)^{-\alpha}$, 


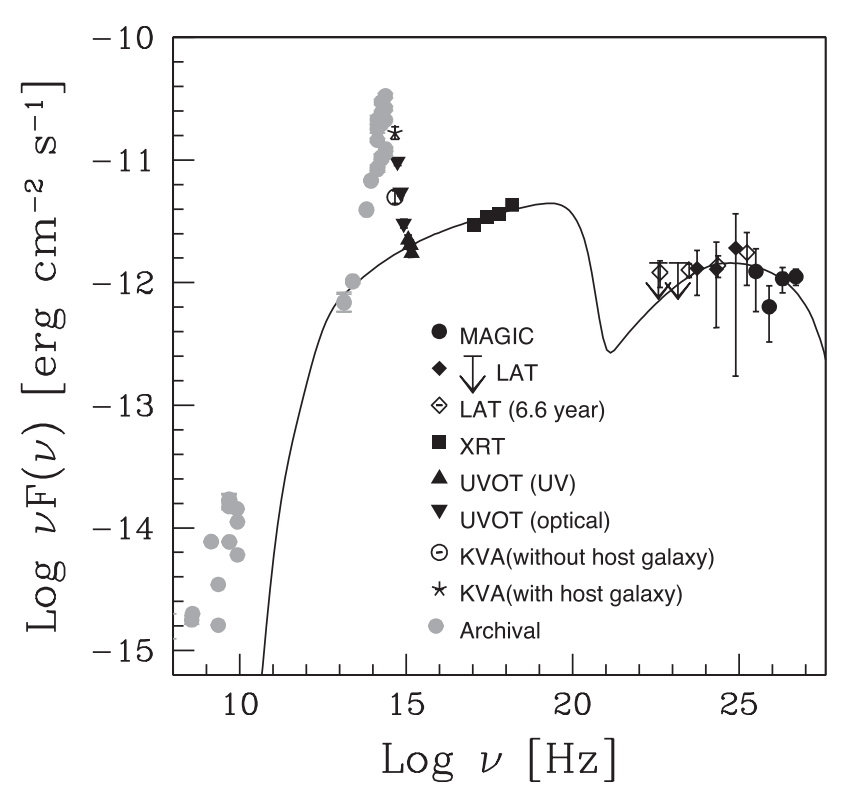

Figure 6. The SED of 1ES $1741+196$ from $\mathrm{eV}$ to $\mathrm{TeV}$ energies. The UV (UVOT UVW1, UVM2, UVW2: filled triangle) fluxes attributed to the nuclear region of blazar are used for the SED fitting - whereas the optical fluxes (KVA: empty circle; UVOT V, B, U: filled inverted triangle) are not. The UVOT UV fluxes are corrected for both the Galactic extinction and the host galaxy, while UVOT optical fluxes are corrected only for the former. The KVA flux with (empty circle) and without (star) subtracting the host galaxy magnitude are also shown. X-ray data (filled square) come from Swift/XRT, averaged over two distinct observations. Contemporaneous (filled diamond) and $6.6 \mathrm{yr}$-integrated (empty diamond; for comparison) HE $\gamma$-ray data come from Fermi/LAT. MAGIC data (filled circle) are EBL corrected (Franceschini et al. 2008). We also show the archival data (filled grey circle) for comparison.

with a photon index of $\alpha=2.4 \pm 0.2_{\text {stat }} \pm 0.2_{\text {syst }}$, and a normalization constant at $0.3 \mathrm{TeV}$ of $f_{0}=\left(4.9 \pm 1.1_{\text {stat }} \pm 0.9_{\text {syst }}\right) \times 10^{-12}$ ph $\mathrm{cm}^{-2} \mathrm{~s}^{-1} \mathrm{TeV}^{-1}$. The systematic error on the energy scale is 17 per cent (Aleksić et al. 2012). The mean integrated flux above $100 \mathrm{GeV}$ is $\mathrm{F}(>100 \mathrm{GeV})=\left(6.4 \pm 1.7_{\text {stat }} \pm 2.6_{\text {syst }}\right) \times$ $10^{-12} \mathrm{ph} \mathrm{cm}^{-2} \mathrm{~s}^{-1}$.

\section{DISCUSSION}

The emission of most BL Lac sources is understood in terms of the one-zone SSC model, in which energetic electrons moving inside a magnetized relativistically moving plasma blob emit via the synchrotron and IC scattering mechanisms (e.g. Tavecchio, Maraschi $\&$ Ghisellini 1998). The electron spectrum is often described as a smoothed broken power law.

$N(\gamma)=\left\{\begin{array}{ll}\mathrm{K} \gamma^{-\mathrm{n}_{1}} & ; \gamma_{\min }<\gamma<\gamma_{\mathrm{br}} \\ \mathrm{K} \gamma_{\mathrm{br}}^{\mathrm{n}_{2}-\mathrm{n}_{1}} \gamma^{-\mathrm{n}_{2}} & ; \gamma_{\mathrm{br}}<\gamma<\gamma_{\max }\end{array}\right\}$

where $\gamma_{\min }, \gamma_{\text {br }}$ and $\gamma_{\max }$ are the lowest, break and highest Lorentz factors, $\mathrm{K}$ is the normalization constant, and $\mathrm{n}_{1}$ and $\mathrm{n}_{2}$ are, respectively, the slopes below and above the break. The relativistic boosting is encoded in the Doppler factor $\delta=\left[\Gamma\left(1-\frac{\mathrm{v}}{\mathrm{c}} \cos \theta\right)\right]^{-1}$, where $\Gamma$ is the bulk Lorentz factor and $\theta$ is the viewing angle.

In Fig. 6, we plot the broad-band SED of 1ES 1741+196 using the multiwavelength data described in Section 3. The VHE data are corrected for the EBL using the Franceschini et al. (2008) model. The Fermi/LAT spectrum (HE $\gamma$-ray band), which is contemporane-

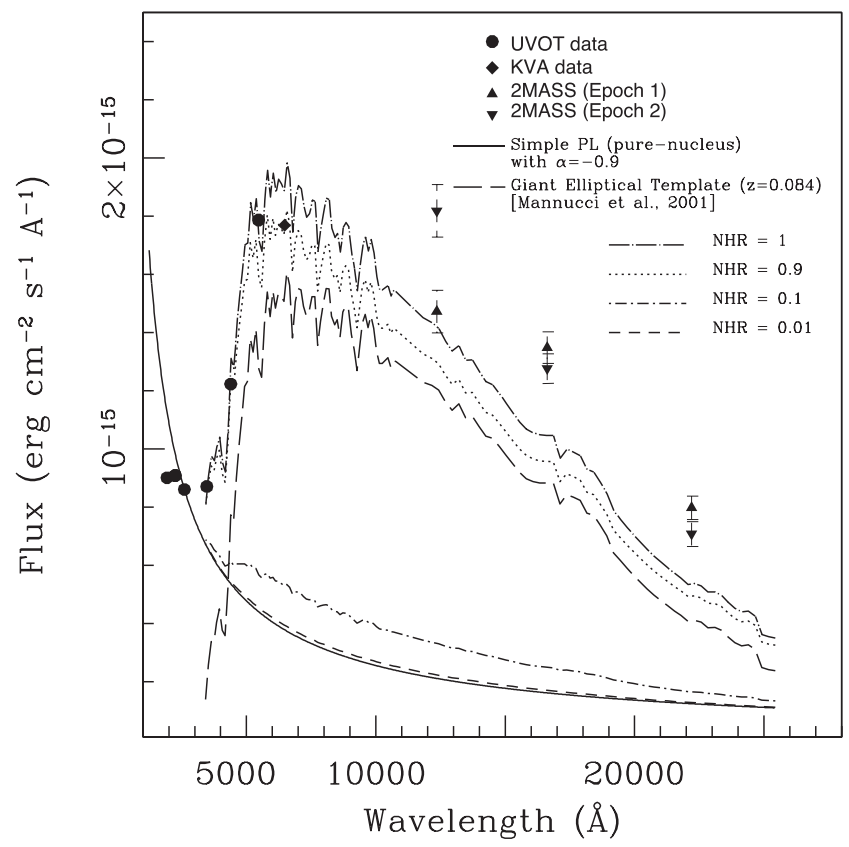

Figure 7. 1ES 1741+196's spectral feature at optical/NIR frequencies, decomposed into blazar and host galaxy components. In the relevant spectral range of interest here, the blazar and the host galaxy are modelled as, respectively, a power law (with a spectral index of 0.9 ) and a giant-elliptical thermal template (with $U-B=0.50, B-V=0.99, V-R=0.59$, $V-I=1.22, V-K=3.30$; see Mannucci et al. 2001).

ous with the MAGIC observation is shown as filled diamonds, while the Fermi spectrum for the total time period (empty diamonds) is not used for the SED modelling. The Swift/XRT X-ray spectrum (averaged over the nights of 2010 July 30 and 2001 Jan 21) is also depicted. The optical KVA point, that represents the blazar's non-thermal optical flux (free from the host galaxy emission), was computed convolving a point-like source with a typical KVA seeing of 2 arcsec.

The SED of 1ES 1741+196 does not look very different from the SEDs of other BL Lacs (Tavecchio et al. 2010). However, it shows an unusual conspicuous feature at optical/NIR frequencies, that appears as a branch taking off from the familiar non-thermal SED. We assume this feature to be the spectral signature of the elliptical galaxy hosting the blazar. In Fig. 7, we overlay the optical/NIR data -Swift/UVOT and KVA - together with the non-simultaneous data from the 2 Micron All Sky Survey (2MASS) obtained on 1999 June $19,{ }^{4}$ on to the giant elliptical template of Mannucci et al. (2001), placed at the appropriate distance: the good agreement below $10000 \AA$ supports our assumption. The difference between the model and the 2MASS flux could be due to the contribution of the two nearby galaxies in the triplet that was not taken into account in this modelling.

We fitted the non-thermal SED using the method described in Mankuzhiyil et al. (2011), i.e. assuming a one-zone SSC emission model (Tavecchio et al. 1998). The SSC model parameters obtained are given in Table 1. Fitting an SSC model to the observed SED data returns parameters that are typically found for HBL (e.g. Tavecchio et al. 2010, Mankuzhiyil et al. 2011, Mankuzhiyil et al. 2012), except for the comparatively lower Doppler factor, and the minor difference between the slopes below and above the $\gamma_{\text {break }}$ of the electron energy

${ }^{4}$ http://www.ipac.caltech.edu/2mass/ 
Table 1. Model parameters used for fitting the SED in Fig. 6.

\begin{tabular}{ccccccccr}
\hline$\gamma_{\min }$ & $\gamma_{\mathrm{br}}$ & $\gamma_{\max }$ & $\mathrm{n}_{1}$ & $\mathrm{n}_{2}$ & $\mathrm{~K}\left(\mathrm{~cm}^{-3}\right)$ & $\mathrm{B}(\mathrm{G})$ & $\mathrm{R}(\mathrm{cm})$ & $\delta$ \\
\hline $1 \times 10^{3}$ & $5.1 \times 10^{3}$ & $9.4 \times 10^{6}$ & 2.2 & 2.9 & $2.3 \times 10^{4}$ & $3.9 \times 10^{-2}$ & $2.0 \times 10^{16}$ & 14.0 \\
\hline
\end{tabular}

distribution (EED). We note that the experimental constraints are relatively limited, hence the SSC parameter combination may not be unique. Alternative combinations of parameters could also provide an acceptable fit to the data.

Our results are in overall agreement with those recently reported by the VERITAS collaboration (Abeysekara et al. 2016), pertaining to observations made after ours. The similar results should not be surprising given that the source is consistent with the hypothesis of no variability during the last $\sim 6 \mathrm{yr}$.

It is interesting to note that the infrared region of the archival data (which were not considered for the SED fit) nicely match with the SED. However, the KVA (host galaxy subtracted) flux does not agree well with the non-thermal SED, while the KVA flux with the host galaxy shows a rough agreement between the non-thermal infrared and optical flux points. The lack of other host-galaxysubtracted data in the optical region prevents us from testing any other emission model (see for example, the helical jet model of Villata \& Raiteri 1999, that was applied in Ahnen et al. 2016).

The radio emission is plausibly from an extended region, hence does not agree with the model below the frequency $10^{11} \mathrm{~Hz}$. As a demonstration, we calculate the typical Lorentz factor $\left(\gamma_{R}\right)$ of electrons that are responsible for the radio emission at $\sim 10 \mathrm{GHz}$ in a magnetic field $3.9 \times 10^{-2} \mathrm{G}$ and Doppler factor 14 (from the Table 1). The calculated value turns out to be $\gamma_{\mathrm{R}} \sim 100$. The cooling time-scale of such an electron will be $t_{\mathrm{c}} \sim 1 . \times 10^{10} \mathrm{~s}$. Assuming, the electron diffuses through the jet medium at the speed of light, the extent of the radio emitting region will be $\sim 100 \mathrm{pc}$, which is far beyond the blob radius. This suggests that the radio is emitted from an extended region, hence may differ from the model.

We note that the Doppler factor from the SSC fit is well above the Doppler factor $(>4)$ calculated from the jet-counterjet radio brightness ratio (Piner \& Edwards 2014). This is a common dispute in blazars, where the Doppler factor from the SSC fit falls mostly in the range of 10-50 (Tavecchio et al. 2010), while it is a few from the radio brightness studies (Piner \& Edwards 2004). This may be because the Doppler factor that we estimate through the SED modelling belongs to the blazar zone. The size of the emission region derived from the SSC fit is $2 \times 10^{16} \mathrm{~cm}$. If we assume a conical jet of opening angle of $1^{\circ}$, then the blazar emission region is located at $\sim 1 \times 10^{18} \mathrm{~cm}$ from the central engine. This distance corresponds to an angular separation of $\sim 0.1$ milliarcsec (at a redshift $z=0.084$ ), which is beyond the resolution of radio telescopes. However, the jet-counterjet brightness ratio is estimated from the extended region of the jet.

Perhaps related to 1ES 1741+196's host galaxy being visible, the equivalent isotropic luminosity, estimated from the peak fluxes and the corresponding frequencies of the synchrotron and SSC components of the SED of this source, $\sim 8.2 \times 10^{43} \mathrm{erg} \mathrm{s}^{-1}$, is among the lowest among TeV blazars. This is at least partly due to its Doppler factor, $\delta \simeq 14$, being lower by a factor of $\sim 2$ than typical values found in TeV blazars - maybe owing to misalignment - as $L \propto \delta^{4}$, this source may indeed appear underluminous by a factor of $\sim 20$.

The $\gamma_{\text {break }}$ of the EED, which lies near the $\gamma_{\text {min }}$, and the minor difference in the EED slopes below and above the $\gamma_{\text {break }}$ (2.2 and 2.9 , respectively) are unusual compared to the EED parameters of other BL Lacs (see for example, Tavecchio et al. 2010, Mankuzhiyil et al. 2011, Mankuzhiyil et al. 2012). The reader may also note that the VERITAS collaboration reported a simple power-law EED (instead of a broken power law that we use) of slope 2.2 to explain the emission process of this source. We have also attempted to fit the SED using a simple power-law EED, and found that the model does not reproduce well the $U V$ band (connected to the X-ray spectrum) and the flatness of the measured high-energy (IC) peak. Therefore, one needs a double power-law EED with an internal break with a relatively small spectral change $\left(\Delta_{n}=0.7\right)$ to describe well the measured broad-band SED reported in this study. The origin of these internal breaks in the EED, presumedly related to the acceleration process, may be due to variations in the global field orientation or turbulence levels sampled by particles of different energy. The need for this kind of internal breaks in the EED have been reported in the literature for several sources in order to better describe the spectral measurements. Examples of those are the ones reported for 3C 454 (Abdo et al. 2009), AO 0235+164 Abdo et al. (2010), Mrk 421 (Abdo et al. 2011a, Aleksić et al. 2015) and Mrk 501 (Abdo et al. 2011b and Mankuzhiyil et al. 2012 during short flares). A detailed study on the emission process will be addressed in a more detailed paper.

To the best of our knowledge, 1ES 1741+196 is the first blazar with known SED hosted in a triplet of interacting galaxies. It is interesting to note that, even though a tidal tail is observed to emanate from the host galaxy (Heidt et al. 1999) - suggesting mass loss from the galaxy due to tidal forces within the triplet - the SSC emission parameters of 1ES $1741+196$ do not substantially deviate from typical values (except the Doppler factor and the slope of EED) observed in other BL Lacs.

\section{SUMMARY}

We reported the first detection (by MAGIC) of VHE $\gamma$-rays from the BL Lac source 1ES 1741+196. From the 2010-2011 MAGIC data the source is clearly detected at $6.0 \sigma$ significance level. The multifrequency data used in this paper suggest that 1ES 1741+196 shows a behaviour typical of HBL sources, with a slightly different EED and a lower Doppler factor. A notable peculiarity of the SED of 1ES $1741+196$ is that it shows the host galaxy's spectral signature, a thermal feature at optical/NIR frequencies that we show is compatible with the spectrum of a giant elliptical. The coincidental relatively low luminosity of 1ES 1741+196 may stem from the jet's relatively low Doppler factor.

\section{ACKNOWLEDGEMENTS}

We would like to thank the Instituto de Astrofísica de Canarias for the excellent working conditions at the Observatorio del Roque de los Muchachos in La Palma. The financial support of the German BMBF and MPG, the Italian INFN and INAF, the Swiss National Fund SNF, the ERDF under the Spanish MINECO (FPA201569818-P, FPA2012-36668, FPA2015-68278-P, FPA2015-69210C6-2-R, FPA2015-69210-C6-4-R， FPA2015-69210-C6-6-R, AYA2013-47447-C3-1-P, AYA2015-71042-P, ESP2015-71662C2-2-P, CSD2009-00064) and the Japanese JSPS and MEXT is gratefully acknowledged. This work was also supported by the Spanish Centro de Excelencia 'Severo Ochoa' SEV-2012-0234 and SEV-2015-0548, and Unidad de Excelencia 'María de Maeztu' MDM-2014-0369, by grant 268740 of the Academy of Finland, by the Croatian Science Foundation (HrZZ) Project 09/176 and the University of Rijeka Project 13.12.1.3.02, by the DFG Collaborative Research Centers SFB823/C4 and SFB876/C3, and by the Polish MNiSzW grant 745/N-HESS-MAGIC/2010/0. The Fermi LAT Collaboration acknowledges generous ongoing support from a number of agencies and institutes that have supported 
both the development and the operation of the LAT as well as scientific data analysis. These include the National Aeronautics and Space Administration and the Department of Energy in the United States, the Commissariat à l'Energie Atomique and the Centre National de la Recherche Scientifique/Institut National de Physique Nucléaire et de Physique des Particules in France, the Agenzia Spaziale Italiana and the Istituto Nazionale di Fisica Nucleare in Italy, the Ministry of Education, Culture, Sports, Science and Technology (MEXT), High Energy Accelerator Research Organization (KEK) and Japan Aerospace Exploration Agency (JAXA) in Japan and the K. A. Wallenberg Foundation, the Swedish Research Council and the Swedish National Space Board in Sweden. Additional support for science analysis during the operations phase is gratefully acknowledged from the Istituto Nazionale di Astrofisica in Italy and the Centre National d'Études Spatiales in France. This research was supported 572 by an appointment to the NASA Postdoctoral Program at the Goddard Space Flight Center, administered by Universities Space Re- 574 search Association through a contract with NASA. F K acknowledges funding from the European Union's Horizon 2020 research and innovation programme under grant agreement No 653477.

\section{REFERENCES}

Abdo A. A. et al., 2009, ApJ, 699, 817

Abdo A. A. et al., 2010, ApJ, 710, 1271

Abdo A. A. et al., 2011a, ApJ, 736, 131

Abdo A. A. et al., 2011b, ApJ, 727, 129

Abeysekara A. U. et al., 2016, MNRAS, 459, 2550

Aleksić J. et al., 2011, ApJ, 729, 115

Acero F. et al., 2015, ApJS, 218, 23

Acero F. et al., 2016, ApJS, 223, 2

Ackermann M. et al., 2012, ApJ, 750, 3

Ackermann M. et al., 2013, ApJS, 209, 34

Ahnen M. L. et al., 2016, MNRAS, 459, 3271

Albert J. et al., 2007, Nucl. Instr. Meth. A, 583, 494

Albert J. et al., 2008a, ApJ, 674, 1037

Albert J. et al., 2008b, Nucl. Instr. Meth., A594, 407

Albert J. et al., 2008c, Nucl. Instr. Meth., A588, 424

Aleksić J. et al., 2012, Astropart. Phys., 35, 435

Aleksić J. et al., 2015, A\&A, 578, A22

Aliu E. et al., 2009, Astropart. Phys., 30, 293

Atwood W. B. et al., 2009, ApJ, 697, 1071

Barthelemy S. D. et al., 2005, Space Sci. Rev., 120, 143

Berger K. et al., 2011, Proc. 32nd ICRC, 8, 115

Burrows D. N. et al., 2005, Space Sci. Rev., 120, 165

Costamante L., Ghisellini G., 2002, A\&A, 384, 56

Dermer C. D., Schlickeiser R., 1993, ApJ, 416, 458

Fomin V. P., Fennell S., Lamb R. C., Lewis c D. A., Punch M., Weekes T. C., 1994, Astropart. Phys., 2, 137

Franceschini A., Rodighiero G., Vaccari M., 2008, A\&A, 487, 837

Fukugita M., Shimasaku K., Ichikawa T., 1995, PASP, 107, 945

Gehrels N. et al., 2004, ApJ, 611, 1005

Heidt J., Nilsson K., Fried J. W., Takalo L. O., Sillanpaa A., 1999, A\&A, 348,113

Hillas A. M., 1985, Proc. 19th ICRC, 3, 445

Kalberla P. M. W., Burton W. B., Hartmann D., Arnal E., Bajaja E., Morras R., Poppel W. G. L., 2005, A\&A, 440, 775

Li T. P., Ma Y. Q., 1983, ApJ, 272, 317

Lindfors E. J. et al., 2016, A\&A 593, A98

Lombardi S. et al., 2011, Proc. 32nd ICRC, 3, 266

Mankuzhiyil N., Ansoldi S., Persic M. Tavecchio F., 2011, ApJ, 733, 14

Mankuzhiyil N., Ansoldi S., Persic M., Rivers E., Rothschild R., Tavecchio F., 2012, ApJ, 753, 154

Mannheim K., 1993, A\&A, 269, 67
Mannucci F., Basile F., Poggianti B. M., Cimatti A., Daddi E., Pozzetti L., Vanzi L.2001, MNRAS, 326, 745

Maraschi L., Ghisellini G., Celotti A., 1992, ApJ, 397, L5

Mattox J. R. et al., 1996, ApJ, 461, 396

Nilsson K., Pasanen M., Takalo L. O., Lindfors E., Berdyugin A., Cipirini S., Pforr J., 2007, A\&A, 475, 199

Padovani P. Giommi P., 1995, ApJ, 444, 567

Piner B. G., Edwards P. G., 2004, ApJ, 600, 115

Piner B. G., Edwards P. G., 2014, ApJ, 797, 25

Rector T. A., Cabzuda D. C., Stocke J. T., 2003, AJ, 125, 1060

Rees M. J., 1967, MNRAS, 135, 345

Roming P. W. A. et al., 2005, Space Sci. Rev., 120, 95

Schlegel D. J., Finkbeiner D. P., Davis M., 1998, ApJ, 500, 525

Tavecchio F., Maraschi L., Ghisellini G., 1998, ApJ, 509, 608

Tavecchio F., Ghisellini G., Ghirlanda G., Foschini L., Maraschi L., 2010, MNRAS, 401, 1570

Villata M., Raiteri C. M., 1999, A\&A, 347, 30

Zanin R. et al., 2011, Proc. 32nd ICRC, 7, 72

${ }^{1}$ ETH Zurich, $\mathrm{CH}-8093$ Zurich, Switzerland

${ }^{2}$ Università di Udine, and INFN Trieste, I-33100 Udine, Italy

${ }^{3}$ Department of Physics, Kyoto University, Kyoto 606-8501, Japan

${ }^{4}$ INAF National Institute for Astrophysics, I-00136 Rome, Italy

${ }^{5}$ Università di Siena, and INFN Pisa, I-53100 Siena, Italy

${ }^{6}$ Università di Padova, and INFN, I-35131 Padova, Italy

${ }^{7}$ Croatian MAGIC Consortium, Rudjer Boskovic Institute, University of Rijeka, University of Split and University of Zagreb, 10000, Croatia

${ }^{8}$ Saha Institute of Nuclear Physics, 1/AF Bidhannagar, Salt Lake, Sector-1, Kolkata 700064, India

${ }^{9}$ Max-Planck-Institut für Physik, D-80805 München, Germany

${ }^{10}$ Universidad Complutense, E-28040 Madrid, Spain

${ }^{11}$ University of Łódź, PL-90236 Lodz, Poland

${ }^{12}$ Deutsches Elektronen-Synchrotron (DESY), D-15738 Zeuthen, Germany

${ }^{13}$ Humboldt University of Berlin, Institut für Physik Newtonstr. 15, D-12489 Berlin, Germany

${ }^{14}$ University of Trieste, Piazzale Europa, 1, I-34127 Trieste, Italy

${ }^{15}$ Institut de Fisica d'Altes Energies (IFAE), The Barcelona Institute of Science and Technology, Campus UAB, E-08193 Bellaterra (Barcelona), Spain

${ }^{16}$ Universität Würzburg, D-97074 Würzburg, Germany

${ }^{17}$ Inst. de Astrofísica de Canarias, E-38200 La Laguna, Tenerife, Spain; Universidad de La Laguna, Dpto. Astrofísica, E-38206 La Laguna, Tenerife, Spain

${ }^{18}$ Institute for Space Sciences (CSIC/IEEC), E-08193 Barcelona, Spain

${ }^{19}$ Technische Universität Dortmund, D-44221 Dortmund, Germany

${ }^{20}$ Finnish MAGIC Consortium, Tuorla Observatory, University of Turku and Astronomy Division, University of Oulu, Finland

${ }^{21}$ Unitat de Física de les Radiacions, Departament de Física, and CERESIEEC, Universitat Autònoma de Barcelona, E-08193 Bellaterra, Spain

${ }^{22}$ Universitat de Barcelona, ICC, IEEC-UB, E-08028 Barcelona, Spain

${ }^{23}$ Japanese MAGIC Consortium, ICRR, The University of Tokyo, Department of Physics and Hakubi Center, Kyoto University, Tokai University, The University of Tokushima, KEK, Japan

${ }^{24}$ Inst. for Nucl. Research and Nucl. Energy, BG-1784 Sofia, Bulgaria

${ }^{25}$ Japanese MAGIC Consortium

${ }^{26}$ INAF - Trieste and Department of Physics and Astronomy, University of Bologna

${ }^{27}$ Università di Pisa, and INFN Pisa, I-56126 Pisa, Italy

${ }^{28}$ ISDC-Science Data Center for Astrophysics, CH-1290 Versoix (Geneva), Switzerland

${ }^{29}$ ICREA and Institute for Space Sciences (CSIC/IEEC), E-08193 Barcelona, Spain

${ }^{30}$ NASA Goddard Space Flight Center, Greenbelt, MD 20771, USA

${ }^{31}$ GRAPPA and Anton Pannekoek Institute for Astronomy, University of Amsterdam, Science Park 904, NL-1098 XH Amsterdam, the Netherlands

${ }^{32}$ ASI Science Data Center, Via del Politecnico snc, I-00133 Roma, Italy

This paper has been typeset from a $\mathrm{T}_{\mathrm{E}} \mathrm{X} / \mathrm{L} \mathrm{T} \mathrm{E}_{\mathrm{E}} \mathrm{X}$ file prepared by the author. 